\title{
Combining RMT-based filtering with time-stamped resampling for robust portfolio optimization
}

\author{
David Quintana ${ }^{1 *}$ Sandra García-Rodríguez ${ }^{2}$ Silvano Cincotti $^{3}$ Pedro Isasi $^{4}$ \\ ${ }^{1}$ Department of Computer Science, Universidad Carlos III de Madrid, \\ Avenida de la Universidad 30, \\ Leganés, Madrid 28223, Spain \\ E-mail:dquintan@inf.uc3m.es \\ ${ }^{2}$ CEA Saclay, DRT/LIST/DM2I/LADIS \\ 91191 Gif-sur-Yvette Cedex, France \\ E-mail: sandra.garciarodriguez@cea.fr \\ ${ }^{3}$ DIME-MIG-CINEF, University of Genoa, \\ Via Opera Pia, 15 \\ 16145 Genova, Italy \\ E-mail: silvano.cincotti@unige.it \\ ${ }^{4}$ Department of Computer Science, Universidad Carlos III de Madrid, \\ Avenida de la Universidad 30, \\ Leganés, Madrid 28223, Spain \\ E-mail: isasi@ia.uc3m.es
}

Received 26 october 2014

Accepted 13 June 2015

\begin{abstract}
Finding the optimal weights for a set of financial assets is a difficult task. The mix of real world constrains and the uncertainty derived from the fact that process is based on estimates for parameters that likely to be inaccurate, often result in poor results. This paper suggests that a combination of a filtering mechanism based on random matrix theory with time-stamped resampled evolutionary multiobjective optimization algorithms enhances the robustness of forecasted efficient frontiers.
\end{abstract}

Keywords: Portfolio optimization, Filtering, Robustness, Multi-objective optimization.

\section{Introduction}

The choice of the right allocation of funds among alternatives is a major topic of interest for both practitioners and researchers focused in finance. The number of alternative distributions funds, portfolios, might be large and their profiles are likely to be dif- ferent. The topic is complex and it can be analysed from different points of view. Bearing that in mind, this work fits in the literature in the specific area of robust portfolio optimization. The intended contribution is an analysis of the potential benefits derived from a combination of a resampled approach based on evolutionary algorithms with a filtering mecha- 
nism derived from random matrix theory.

The amount of research devoted to portfolio optimization is ample and it is mostly based on core contributions made by Markowitz ${ }^{1,2}$. This author frames the problem of asset allocation as multiobjective optimization task where the investor tries to balance two opposing objectives, the minimization of risk and the maximization of return of the portfolio as a whole.

Formally, the basic version of the bi-objective problem could be defined as:

- Minimize portfolio risk:

$$
\sigma_{p}^{2}=\sum_{i=1}^{n} \sum_{j=1}^{n} w_{i} w_{j} \sigma_{i j}
$$

- Maximize portfolio return:

$$
E\left(R_{p}\right)=\Sigma_{i=1}^{n} w_{i} \mu_{i}
$$

- Subject to the following constraints:

$$
\begin{gathered}
\sum_{i=1}^{n} w_{i}=1 \\
0 \leqslant w_{i} \leqslant 1 ; i=1 \ldots n
\end{gathered}
$$

where $n$ is the number of available assets, $\mu_{i}$ the expected return of asset $i, \sigma_{i j}$ the covariance between asset $i$ and $j$, and $w_{i}$ are the weights for the components of the portfolio. These weights are the decision variables. For practical purposes, risk is represented with the standard deviation $\sigma_{p}$. The constrains referenced in equations 3 and 4 represent requirements for the full investment of funds and the prohibition from shorting any asset, respectively.

This framework may be extended to consider some additional real world constraints ${ }^{3}$ such as:

- Cardinality constraint: it is possible to define the maximum $C_{\max }$ and minimum $C_{\min }$ number of assets in which it is possible to invest $\left(w_{i} \neq 0\right)$ :

$$
C_{\text {min }} \leqslant \Sigma\left(w_{i} \neq 0\right) \leqslant C_{\text {max }}
$$

- Values limit constraint: each weight $w_{i}$ must have a value in the interval $\left[\lim _{\text {inf }}, \lim _{\text {sup }}\right]$, where:

$$
0.0 \leqslant \lim _{\text {inf }} \leqslant w_{i} \leqslant \lim _{\text {sup }} \leqslant 1.0
$$

The solution for this problem consists of a set of portfolios that defines a Pareto front, which is usually referred to as the efficient frontier. The points of this curve represent portfolios that have the minimum amount of risk given a certain expected return, and viceversa. Given that the preference for one objective over the other one is conditioned by the attitudes of the decision maker, these portfolios are neither better or worse than the rest of the components of the solution. These portfolios, plotted in the risk-return space, define a curve similar to fig. 1.

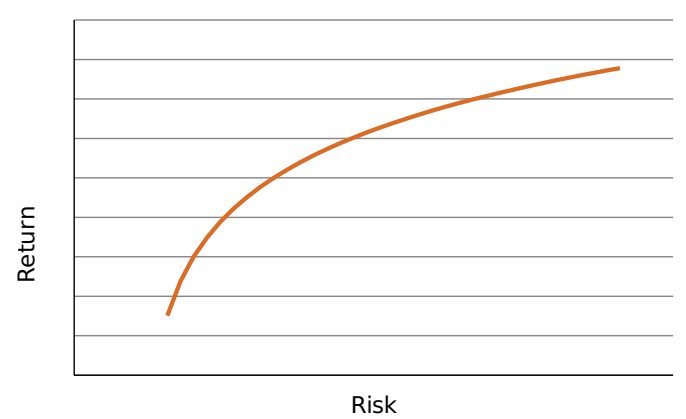

Fig. 1. Efficient frontier

The difficulty of tackling this optimization problem varies widely depending on the assumptions. Once the basic model is extended with real world ones, the limitation of standard exact approaches becomes apparent. For this reason, both academics and practitioners have been exploring for decades the possibilities offered by evolutionary computation.

The multiobjective nature of the problem makes it well suited for Multiobjective Evolutionary Algorithms (MOEAs). As reflected in a paper by Metaxiotis and Liagkouras ${ }^{4}$, there is a large number of recent papers exploring their performance in portfolio optimization. Among them, we could mention Skolpadungket et $a .^{5}$, Radziukyniene and Xilinskas ${ }^{6}$, Anagnostopoulos and Mamanis ${ }^{7}$ or Deb et al. ${ }^{8}$.

Despite all this research effort on MOEAS for portfolio optimization there are still open issues that require further investigation such as the problem of robustness ${ }^{9}$. The lack of robust solutions is one of the main concerns regarding optimization; in consequence some approaches have emerged that deals with that issue ${ }^{10}$. Focusing on asset allocation, we find that the reliability of the solutions provided by 
the algorithms is subject to the accuracy of the estimates for two key parameters that underlie the optimization process: the expected asset returns and their associated variance-covariance matrix. Their true values are difficult to predict, and this often results on expected efficient frontiers that turn out to lie far from the observed ones.

The problem of finding robust portfolios has already been studied in literature ${ }^{11}$. Among the different authors who have tackled this issue we could mention Costa et al. ${ }^{12}$ who deals with the problem of robust optimal portfolio selection, or Nguyen et al. ${ }^{13}$ who introduce a generic robust ranking model and apply it to portfolio optimization. Literature shows some other studies which work in the optimization of the worst-case scenarios such as Ghaoui et al. ${ }^{14}$, who consider the problem of computing and optimizing (by using conic optimization) the worstcase mean-variance, or Zymler et al. ${ }^{15}$, who suggest a novel robust optimization model for designing portfolios, that model trades off weak and strong guarantees on the worst-case portfolio return.

Summarizing, most of the literature on robust portfolio optimization falls into two fields: papers that emphasize the need for robust estimates for the parameters or those where authors try to manage uncertainty in the optimization process itself. The first one usually tries to filter the estimates to control, for instance, the influence of extreme past events on their computation ${ }^{16}$. We also find many authors who focus on a second way, handling the uncertainty in parameters during the optimization process $17,18,19,20$. Other efforts regarding the second line were made to introduce robustness in multiobjective optimization ${ }^{21}$. For instance Deb et al. ${ }^{22}$ launched a robust adaptation for NSGA-II algorithm to optimize portfolios and then Garcia et al. ${ }^{23}$ dealt with the problem of robustness but using resampling and a third objective as a time-stamp.

In this paper we suggest combining both, timestamped resampling and filtering the variancecovariance matrix or, more exactly, the associated correlation matrix. Details of this new approach will be provided in the next section. Then, a wide set of experiments is shown in order to validate the approach. Finally, the last section will be focused on conclusions and future work.

\section{Robust Approach}

As we already discussed in the introduction, the portfolio optimization process depends on imperfect forecasts for two key sets of parameters, future returns and the variance-covariance matrix. Given that this usually has a major impact in the reliability of the results, there is a clear need for control mechanisms. In this section we introduce two approaches that have never been tested together: a set of filters based on random matrix theory and time-stamped resampling strategy based on MOEAs. This will be followed by an introduction to robustness metrics.

\subsection{RMT Filtering}

The origins of Random Matrix Theory (RMT) can be traced back to efforts by nuclear physicists during 50 's and 60's in the context of gaining understanding the energy levels of complex nuclei. More recently, these studies on the statistical properties of matrices based on independent random elements have been tested on the context of portfolio optimization by the econophysics community $24,25,26,27$.

These authors, among others, show that empirical variance-covariance matrices of financial returns are affected by spurious correlations. The cross-correlation matrix, directly connected to the variance-covariance matrix, shows a mix of noise, correlation among the random component of the returns series, and signal. As Laloux et al. report ${ }^{25}$, the consequence of using the correlation matrix directly obtained from historical data is an underestimation of real risk. Hence, the need of using a corrected one.

RMT provides the tools to identify the mentioned two components analysing the eigenvalues, $\lambda$, of the correlation matrix. Given that theoretical limits for the distribution of eigenvalues for random matrices are known ${ }^{28}$, it is possible to identify most of purely random component. Plerou et al. ${ }^{24}$ review the original analytical results and conclude that for return series where the number of assets, $n$, is larger than the number of time periods considered, $p$, the 
lower limit, $\lambda_{\min }$, and the upper one $\lambda_{\max }$, are given by the analytical expression:

$$
\lambda_{\max / \min }=1+\frac{1}{q} \pm 2 \sqrt{\frac{1}{q}}
$$

where $q=p / n$.

These authors suggest a reconstruction process where the original noisy correlation matrix, $C$, is replaced by a filtered one. It starts with the construction of a diagonal matrix $\Lambda$, with $n$ eigenvalues for $C$. Then, those values in the range defined by equation 7 are replaced with zeros and the resulting structure, $\Lambda^{\prime}$, gets transformed to the basis of $C$. Using the eigendecomoposition theorem with the original eigenvectors $E$ we would obtain the filtered cross-correlation matrix $C^{\prime}$ using the expression

$$
C^{\prime}=E \Lambda^{\prime} E^{-1}
$$

The final step would be setting the diagonal elements of $C^{\prime}$ to 1 in order to make sure that both $C$ and $C^{\prime}$ have the same trace. The outcome of all this is a clean correlation matrix that can subsequently be used in the optimization process.

While different authors suggest different approaches to get to $C^{\prime}$, the core ideas are the same. They tend to differ only in the choice of the values used to replace the eigenvalues in the range $\lambda_{\min / \max }$ in $\Lambda$ to get $\Lambda^{\prime}$.

Daly et al. ${ }^{27}$ review several filtering approaches and introduce a new one based on Krzanowski stability ${ }^{29}$. The method is very similar to the one described above. The difference is that the replacement eigenvalues for $\Lambda^{\prime}$ are given by the expression

$$
\lambda_{i}^{\prime}=\lambda_{1}^{\prime}+(i-1) k
$$

In this context, $k$ is a constant defined as

$$
k=2 \frac{a-\lambda_{1}^{\prime}}{r-1}
$$

where $r$ is the number of noisy eigenvalues to be replaced and $a$ is their mean $\left(\bar{\Lambda}_{\text {noisy }}\right)$.

The key parameter to be defined is the minimum value $\lambda_{1}^{\prime}$, which the authors define in terms of a fraction of $a$. KR2, therefore, would correspond to a Krzanowski stability-based filter with $\lambda_{1}^{\prime}=a / 2$, KR4 to a filtering with $\lambda_{1}^{\prime}=a / 4$ etc.

Daly et al. report that, in their experiments, KR2, KR4 and KR8 showed the highest potential for realised risk reduction. For this reason, we will consider them, together with the RMT filtering by Plerou et al., in our experimental analysis. The filtering methods described above will be combined with a time-stamped resampled optimization strategy

\subsection{Optimization Algorithm}

The multiobjective nature of the optimization problem to be solved, together with the complexity derived from the real-world constraints, makes strategies based on MOEAs very appropriate. A basic modelling would provide approximations to the efficient frontier that would suffer from the unreliability discussed before, hence our choice of complementing them with a time-stamped resampling strategy.

This approach, proposed by García et al. ${ }^{23}$, generates sets of likely scenarios, pairs of expected returns and variance-covariance matrices, based on historical data following the nonparametric bootstrap process described in algorithm $1^{23}$. Each candidate portfolio faces a number of scenarios during the evolution process and the algorithm weeds out those that are too sensitive to deviations in expected returns and variance-covariance matrix. This general process limits the possibility of selecting portfolios that are overspecialized in a scenario, the expected asset returns and the forecasted variancecovariance matrix, that will differ from reality with a high probability. The algorithm also considers the age of candidate portfolios, and favors those that have performed better for a longer time. This timestamped component of the approach, together with the resampling, improves the robustness of the solu- 
tions.

Algorithm 1: Resampling method

1: $S$ : original sample set with a size $N_{s}$.

2: $S^{\prime}$ : new sample set with a size $N_{s}^{\prime}$. At the beginning, $S^{\prime}=\varnothing$ and $N_{s}^{\prime}=0$.

3: while $N_{s}^{\prime} \neq N_{s}$ do

4: $\quad$ Select one instance $X_{i}$, at random, from $S$.

5: Add instance to the new set. $S^{\prime}=S^{\prime}+X_{i}$.

6: end while

7: return $\left(S^{\prime}\right)$

Given that the combination of RMT filtering and time-stamped resampling that we suggest is compatible with many MOEAS, we will test the approach with four of the most popular: NSGA-II, SPEA2, GDE3 and SMPSO. These metaheuristics represent three different algorithm families, genetic algorithms, differential evolution and particle swarm optimization, and we introduce them next.

Genetic algorithms have two representatives in the set. The first one is the Non-dominated Sorting Genetic Algorithm II, NSGA-II, proposed by Deb et al. ${ }^{30}$. It is a multi-objective genetic algorithm that operates applying the standard genetic operators (selection, crossover, and mutation) to a basic population. Then, it sorts both the individuals in the new and old populations according to a dominance rank. The best solutions are chosen to create a new population. In case of tie in terms of rank, the algorithm considers a density estimation based on the crowding distance to the surrounding individuals belonging to the same rank. The second one is the Strength Pareto Evolutionary Algorithm 2, SPEA2. It was proposed by Zitler et al. ${ }^{31}$ and one of its core features is that each individual has a fitness value that is the sum of its strength raw fitness plus a density estimation. It also uses a population of solutions plus an external archive. The algorithm applies the mentioned standard operators to a population of solutions to fill the archive of individuals; then, the nondominated individuals from the combination are copied into a new population. If the number of nondominated individuals happens to be greater than the population size, a truncation operator based on the distances to the $k$-th nearest neighbor is used.

Multiobjective particle swarm optimization al- gorithms are represented by the Speed-constrained Multi-Objective PSO algorithm, SMPSO ${ }^{32}$. In PSO algorithms a set (swarm) of candidate solutions (particles) to the problem navigate through the solution space. This navigation is guided by a velocity equation, which specifies the way particles update their location. Two key elements in this equation are the current position of the particle and the best positions visited so far. Usually, both the best position visited by the particle and the best position visited by any particle in the swarm are taken into account. The algorithm uses an external archive to store nondominated solutions and extends the basic framework adding a constraining mechanism, already applied in mono-objective PSO algorithms, that modulates the speed at which particles fly ${ }^{33}$.

Finally, we will consider differential evolution by means of the Generalized Differential Evolution 3 algorithm, GDE3 ${ }^{34}$. This alternative starts with a population of random solutions, which becomes current population. Every generation, the algorithm extends current population with an offspring population obtained using the differential evolution operators. The result is subsequently reduced to maintain the size stable using nondominated sorting and a pruning technique aimed at diversity preservation.

For this work, in order to ensure fair comparisons, we will use the same encoding and repair operator across core algorithms. Portfolios will be represented by arrays of as many floats as available investment alternatives, where $w_{i}$, represents the percentage invested in asset $i$. Solutions would consist of sets of nondominated portfolios in terms of risk and return that approximate the efficient frontier. As for the repair operator, the need to meet the set of hard constraints (Eqs. 3 to 6) requires a way to deal with unfeasible solutions. The approach to be used will be relying on the operator both after the initialization of the population and the use of the genetic operators.

Whenever the number assets where $w_{i}>0$ is out of the interval $\left[C_{\min }, C_{\max }\right]$, the individual goes through an adjustment process to ensure compliance with the cardinality constraint. This is done adding or dropping assets until the requirement is met. In case the sum of weights per individual is not 
$1.0, \sum_{i=1}^{n} w_{i} \neq 1$, the algorithm adjusts the holdings adding or subtracting random amounts up to the required adjustment. These processes are described in detail in 23 .

\subsection{Robustness Evaluation}

The evaluation of robust solutions requires specific quality indicator that differ for the the most commonly used on in multiobjective optimization such as Hypervolume (HV) or Spread ${ }^{35}$. We will consider four indicators to measure robustness that mostly capture the divergence between expected results and actual results. These are Estimation Error, Stability, Unrealized Returns, and Extreme Risk ${ }^{23}$. We outline their main features.

The first one to be considered is Estimation Error. This indicator measures the average Mahalanobis distance ${ }^{36}$ between the expected risk and return for every portfolio in the efficient frontier and the actual risk and return a posteriori, once the real values of the parameters are observed. As a result, the smaller the value, the closer that the expected behaviour of the optimized portfolios would be to the real one observed a posteriori.

The second quality indicator is Stability. In this case, metric measures the average difference between the Pareto front obtained for the expected scenario, and $S$ feasible alternatives generated using the nonparametric bootstrap described in algorithm 1 . Given that the use of a large set of scenarios is likely to result on a good approximation to the real potential distribution of parameters, high values of this metric would imply higher sensitivity to likely deviations form the expected scenario and, therefore, lower reliability.

The Extreme Risk metric is closely connected to the previous one. Both share the same definition, but they differ in the set scenarios that is considered. In this case, it considers the behaviour of the portfolios in the solution when they face the worst-case scenarios. These are are defined as the $w$ resampled scenarios, out of a $S$ sample, with the highest average Mahalanobis distance between the risk/return of the portfolios evaluated with these parameters and the expected risk/return of the same portfolios. The higher the metric, the lower the robustness.
Finally, Unrealized Returns evaluates, for every portfolio in the solution, the difference between its realized return and the maximum potential return for that risk level. It represents potential income left on the table. Hence, the higher the value for this indicator, the larger the unrealized potential returns. This meas a low value for this indicator would be considered desirable.

\section{Experimentation}

The combined approach described before was tested with historical financial data using the four mentioned core algorithms: NSGA-II, SPEA2, GDE3 and SMPSO. In this section we report details on the experimental design, sample used, parametrization and results.

\subsection{Experimental Setup}

The approach was tested on a set of historical returns for the components of the Spanish broad financial index IBEX-35. The experiments were performed using a sample of 240 monthly returns, 20 years worth of data, covering the period from January 1994 to December 2013. Given that the composition of the index changed during the period, we selected the companies in the index as of the end of 2013 for which the was complete data. That meant that original set of 35 companies was reduced to 18 . The source for the data was the commercial provider Datastream.

The cardinality constraints $\left[C_{\min }, C_{\max }\right]$ and the limits to the minimum and maximum weight that each asset can potentially have in the portfolios that are part of the solution $\left[\lim _{\text {inf }}, \lim _{\text {sup }}\right]$, were set to $[2,16]$ and $[0.1,0.8]$ respectively.

In order to assure that the algorithms face a wide range of historical situations, we decided to use a sliding window approach. This means that the algorithm relies on data from $t_{1}$ to $t_{n}$ to identify the best possible allocations for the period $t_{n+1}$. For this purpose, the size of the mentioned window was set to $n=120$ return periods, that is, to 10 years of data. Therefore for each experiment the 10-year window will move one month, 120 times in total. It is worth noting that this is not a single testing ground for 
a dynamic multi-period approach over 120 months, but a set of 120 different single-period portfolio optimization problem instances. Given the stochastic nature of the algorithms used, all the experiments were repeated 30 times.

The implementation was made in jMetal ${ }^{37}$. This Java framework, designed for multiobjective optimization includes several different metaheuristics. By reusing the base classes of jMetal we ensure a fair comparison as all the alternatives share the same basic core components (solution encodings, operators, etc.). Table 1 shows the parameters used for each algorithm. These have not been optimized and are the default values provided in the framework.

Table 1. Parameters. $\mathrm{L}=18$ (size of individuals). The termination condition is performing 300 iterations.

\begin{tabular}{l|l}
\hline \multicolumn{2}{c}{ SPEA2 } \\
\hline $\begin{array}{l}\text { Population size } \\
\text { Archive size }\end{array}$ & 200 individuals \\
$\begin{array}{l}\text { Crossover } \\
\text { Mutation }\end{array}$ & SBX, $p_{c}=0.9$ \\
Selection of Parents & Polynomial, $p_{m}=1 / \mathrm{L}$ \\
\hline \multicolumn{2}{c}{ NSGA-II } \\
\hline $\begin{array}{l}\text { Population size } \\
\text { Crossover }\end{array}$ & SBO individuals \\
Mutation & Polynomial, $p_{m}=1 / \mathrm{L}$ \\
Selection of Parents & Binary tournament \\
\hline \multicolumn{2}{c}{ SMPSO } \\
\hline $\begin{array}{l}\text { Archive size } \\
\text { Swarm size }\end{array}$ & 200 particles \\
Mutation & 200 particles \\
\hline \multicolumn{2}{c}{ Polynomial, $p_{m}=1 / \mathrm{L}$} \\
\hline Population size & 200 individuals \\
Crossover & DE crossover, CR $=0.9$ \\
Mutation & DE mutation, $F=0.5$ \\
Selection of Parents & DE selection \\
\hline
\end{tabular}

Regarding the parameters needed to compute the metrics, the number of scenarios $S$ necessary to compute Stability and Extreme Risk will be set to 500. The second indicator would also require the specification the percentage of worst-case scenarios, out of those 500 , to be considered. In this case, the metric will be based on the worst $1 \%$, that is, $w=5$.

\subsection{Results}

The combined approach was tested on the four MOEAS already introduced (SPEA2, NSGA-II,
GDE3 and SMPSO) using four different filters: KR2, KR4, KR8 plus the one based of Plerou et al.. The robustness of the solutions was assessed using EE, ST, ER and UR. For each of these metrics, we report the average, median and variance obtained with every setup over the 30 runs in tables 2 to 5 . We also report the results for the basic algorithms and tested the statistical significance of the differences between the basic algorithm and the robust versions. Due to the lack of normality of the distribution, we relied on the Wilcoxon test.

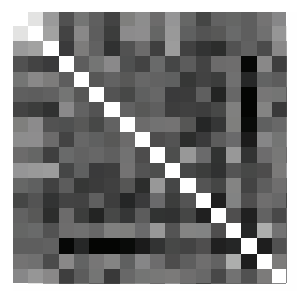

(a) Base

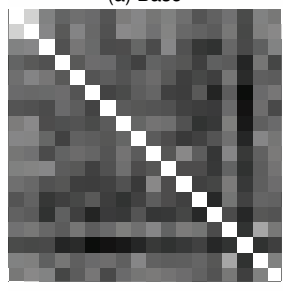

(c) KR4

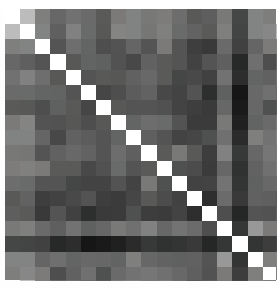

(b) KR2

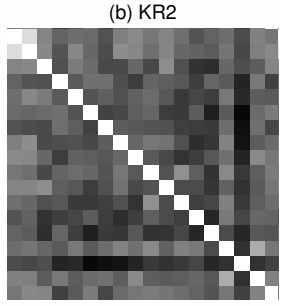

(d) KR8
Fig. 2. Correlation matrices. Base vs KR filtering.

The effect of KR2, KR4 and KR8 filters on the correlation matrix is illustrated in figure 2. This panel shows a graphic representation of correlation matrices in gray-scale. Given that we have 18 investment alternatives, each graph consists of a $18 \times 18$ symetric grid. The lighter the color, the larger the number, hence the fact that the main diagonal, where the correlations are always equal to one, is white. This way, we can observe the differences between the original noisy matrix and the filtered versions. In this example, the underlying data corresponds to the first time period considered in the sliding window, that is, returns from January 1994 to December 2003. As we can see, the cross correlations of the returns for the 18 companies tend to be low. We can infer from the graphs that KR2 filtering affects the original matrix to a higher degree than the rest. Among the other two, KR8 reconstruction leads to more subtle changes. 


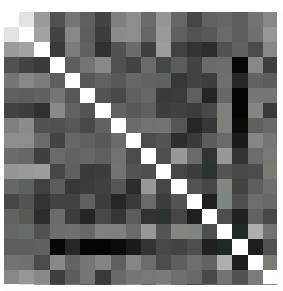

(a) Base

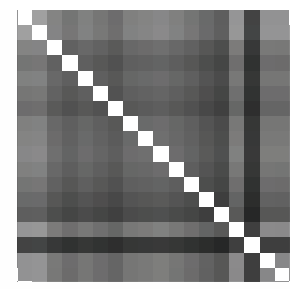

(b) PL
Fig. 3. Correlation matrices. Base vs Plerou et al. filtering.

Figure 3 is very similar. It compares side-byside the original correlation matrix with the reconstructed one once we used the approach by Plerou et al. In this case, the scale of the differences in the color pattern makes apparent that this second strategy leads to changes of a larger magnitude than the suggested KR alternatives.

Table 2. ST - Stability metric.

\begin{tabular}{lrrr}
\hline SPEA2 & Average & Median & Variance \\
\hline Base & 3.9767 & 3.5364 & 3.3538 \\
Combined KR2 & 2.2052 & 1.9873 & 0.7706 \\
Combined KR4 & 2.2154 & 2.0039 & 0.7776 \\
Combined KR8 & 2.2144 & 2.0080 & 0.7656 \\
Combined PL & 2.2159 & 2.0007 & 0.7780 \\
\hline \hline NSGAII & Average & Median & Variance \\
\hline Base & 3.9771 & 3.5246 & 3.3746 \\
Combined KR2 & 2.1250 & 1.9601 & 0.6073 \\
Combined KR4 & 2.1161 & 1.9395 & 0.6118 \\
Combined KR8 & 2.1144 & 1.9537 & 0.6008 \\
Combined PL & 2.1494 & 1.9631 & 0.6131 \\
\hline \hline GDE3 & Average & Median & Variance \\
\hline Base & 3.2202 & 2.9744 & 1.3919 \\
Combined KR2 & 2.5142 & 2.2699 & 1.0856 \\
Combined KR4 & 2.5242 & 2.2935 & 1.0553 \\
Combined KR8 & 2.5241 & 2.2910 & 1.0459 \\
Combined PL & 2.5241 & 2.2961 & 1.0371 \\
\hline \hline SMPSO & Average & Median & Variance \\
\hline Base & 3.5175 & 3.2155 & 1.6320 \\
Combined KR2 & 2.3057 & 2.1029 & 0.7250 \\
Combined KR4 & 2.3055 & 2.1090 & 0.7431 \\
Combined KR8 & 2.3064 & 2.1258 & 0.7174 \\
Combined PL & 2.3301 & 2.1410 & 0.7686 \\
\hline
\end{tabular}

Table 2 shows the main descriptive statistics for the Stability robustness indicator. In terms of stability, NSGA-II is the core algorithm that obtains the best values for both the metric and the largest average improvement. The Daly et al.-based KR filters combined with the time-stamped resampled ap- proach offer the best performance for all the core algorithms with the exception of GDE3. The results for the same filters are similar, but the filter described by Plerou et al. tends to offer slightly worse results. Combined KR8 over NSGA-II improves the basic value of the metric by $46.8 \%$. It is worth noting that the combined approach offers, in addition to a lower mean value for the metric, a clearly smaller variance.

Table 3. EE - Estimation Error metric.

\begin{tabular}{lrrr}
\hline SPEA2 & Average & Median & Variance \\
\hline Base & 1.2068 & 0.8152 & 1.2250 \\
Combined KR2 & 0.8843 & 0.7421 & 0.5085 \\
Combined KR4 & 0.8742 & 0.7461 & 0.5087 \\
Combined KR8 & 0.8664 & 0.7340 & 0.4984 \\
Combined PL & 0.9035 & 0.7470 & 0.5435 \\
\hline \hline NSGAII & Average & Median & Variance \\
\hline Base & 1.2038 & 0.8630 & 1.0827 \\
Combined KR2 & 0.8541 & 0.7090 & 0.4956 \\
Combined KR4 & 0.8402 & 0.6923 & 0.4920 \\
Combined KR8 & 0.8412 & 0.6926 & 0.4854 \\
Combined PL & 0.8787 & 0.7078 & 0.5409 \\
\hline \hline GDE3 & Average & Median & Variance \\
\hline Base & 1.2314 & 1.0071 & 0.7354 \\
Combined KR2 & 1.0840 & 0.9054 & 0.6934 \\
Combined KR4 & 1.0777 & 0.9086 & 0.6717 \\
Combined KR8 & 1.0788 & 0.9177 & 0.6885 \\
Combined PL & 1.1398 & 0.9472 & 0.8058 \\
\hline \hline SMPSO & Average & Median & Variance \\
\hline Base & 1.2943 & 1.0869 & 0.7762 \\
Combined KR2 & 1.0137 & 0.8998 & 0.5291 \\
Combined KR4 & 1.0131 & 0.8751 & 0.5268 \\
Combined KR8 & 1.0048 & 0.8756 & 0.5046 \\
Combined PL & 1.0426 & 0.9122 & 0.5432 \\
\hline & & &
\end{tabular}

The indicator that captures the discrepancy between the expected behavior of the selected portfolios and reality, EE, shows clear pattern of improvement across basic algorithms and filtering strategies. Having said that, the contribution of the combined approach is more variable and tends to be relatively lower if we compare it to the previous metric. Out of the four basic algorithms, NSGA-II provides the best starting point with an average error of 1.2 (very close to the result of the naked version on SPEA2). The combined robust approaches also tend to be specially appropriate with this algorithm as it offers the lowest average value for the metric. In this case, the improvement in relative terms is not as sizeable as it 
was for ST. PL filtering clearly offers results that are worse that KR alternatives. KR4 and KR8 tended to offer the best results. The former one with NSGA-II and GDE3, and the latter with SMPSO and SPEA2.

Table 4 shows the results regarding the sensitivity of the solution to a set of worst-case scenarios where the deviations in terms of risk and return are especially relevant, the robustness indicator labeled Extreme Risk. Given that ER and EE are so closely linked, it not surprising that they show a similar behavior. Once again, the combined approach improves the robustness of the basic algorithms across the board, but the degree to which these algorithms benefit varies. NSGA-II offers the best results followed by the other genetic algorithm, SPEA2, SMPSO and GDE3. The best combinations were also consistent and KR4 and KR8 resulted in lower values for the metric that PL.

Table 4. ER - Extreme Risk metric.

\begin{tabular}{lrrr}
\hline SPEA2 & Average & Median & Variance \\
\hline Base & 1.5884 & 1.2105 & 1.2846 \\
Combined KR2 & 1.2174 & 1.0547 & 0.6234 \\
Combined KR4 & 1.2014 & 1.0504 & 0.6028 \\
Combined KR8 & 1.2000 & 1.0404 & 0.6026 \\
Combined PL & 1.2087 & 1.0600 & 0.6668 \\
\hline \hline NSGAII & Average & Median & Variance \\
\hline Base & 1.5944 & 1.2877 & 1.1464 \\
Combined KR2 & 1.1823 & 1.0095 & 0.6262 \\
Combined KR4 & 1.1710 & 1.0011 & 0.6077 \\
Combined KR8 & 1.1746 & 1.0132 & 0.6037 \\
Combined PL & 1.1866 & 1.0155 & 0.6839 \\
\hline \hline GDE3 & Average & Median & Variance \\
\hline Base & 1.6226 & 1.4283 & 0.9005 \\
Combined KR2 & 1.4616 & 1.2669 & 0.9286 \\
Combined KR4 & 1.4484 & 1.2774 & 0.8906 \\
Combined KR8 & 1.4489 & 1.2697 & 0.9054 \\
Combined PL & 1.4813 & 1.2649 & 0.9846 \\
\hline \hline SMPSO & Average & Median & Variance \\
\hline Base & 1.6961 & 1.5109 & 0.8972 \\
Combined KR2 & 1.4381 & 1.2716 & 0.7167 \\
Combined KR4 & 1.4425 & 1.2509 & 0.7200 \\
Combined KR8 & 1.4350 & 1.2503 & 0.7055 \\
Combined PL & 1.4575 & 1.2724 & 0.7765 \\
\hline
\end{tabular}

The combined approach also has positive impact on the divergence between the Pareto front defined by the selected portfolios and the actual observed efficient frontier once the key parameters were known. Table 5 shows that the addition of the combined ap- proach designed to increase robustness reduces the amount of money left of the table for all cases. The best results were obtained with SMPSO. The multiobjective PSO, combined with the time-stamped resampling and a KR8 filtering achieved a $34.7 \%$ improvement over the basic SMPSO. As we can see in the variances, the algorithm also turns out to be, by far, the most consistent alternative among algorithm runs. Consistently with the rest of the quality indicators, the performance of PL filtering was slightly worse that the Daly et al.-based KR alternatives

Table 5. UR - Unrealized Returns metric.

\begin{tabular}{lrrr}
\hline SPEA2 & Average & Median & Variance \\
\hline Base & 8.4864 & 7.3674 & 24.3744 \\
Combined KR2 & 5.9254 & 5.3010 & 8.6752 \\
Combined KR4 & 5.9204 & 5.2848 & 8.6298 \\
Combined KR8 & 5.8911 & 5.2439 & 8.4073 \\
Combined PL & 5.9489 & 5.3033 & 8.8232 \\
\hline \hline NSGAII & Average & Median & Variance \\
\hline Base & 8.4063 & 7.4374 & 22.6234 \\
Combined KR2 & 5.8588 & 5.2758 & 8.3047 \\
Combined KR4 & 5.8228 & 5.2553 & 8.0605 \\
Combined KR8 & 5.8288 & 5.2678 & 8.0573 \\
Combined PL & 5.9400 & 5.3924 & 8.9086 \\
\hline \hline GDE3 & Average & Median & Variance \\
\hline Base & 6.2664 & 5.6185 & 12.3380 \\
Combined KR2 & 4.7809 & 4.1935 & 9.5771 \\
Combined KR4 & 4.7840 & 4.2502 & 8.9486 \\
Combined KR8 & 4.7521 & 4.1845 & 8.8256 \\
Combined PL & 4.7894 & 4.2482 & 9.1235 \\
\hline \hline SMPSO & Average & Median & Variance \\
\hline Base & 6.6458 & 5.8349 & 13.3521 \\
Combined KR2 & 4.3572 & 3.8316 & 5.3117 \\
Combined KR4 & 4.3420 & 3.8478 & 5.4500 \\
Combined KR8 & 4.3850 & 3.8172 & 5.4756 \\
Combined PL & 4.3859 & 3.8898 & 5.2117 \\
\hline
\end{tabular}

The results of the experimentation suggest that the combined strategy improves the robustness of the solutions. The gain obtained depends on the metric and core algorithm, but the final effect is always positive. The choice of the filtering mechanism affects the results, and there is a clear pattern that shows that KR filtering contributes to enhance robustness to a greater degree than PL. Among KR options, KR4 and KR8 generally are the best alternatives. Despite these differences, in every instance the combined approach led to sizable improvements in all metrics with respect to the non-robust standard 
algorithms. All the reported median differences between the baseline values of the metric for the standard MOEAs and the robust versions were significant at $1 \%$.

\section{Conclusions}

The issue of robustness is one of the most important open research lines in financial portfolio optimization. The fact that traditional methods tend to be very sensitive to deviations on the estimates for future returns and the associated variance-covariance matrix often leads to unreliable forecasts for the efficient frontier. For this reason, in this paper we suggested using a combined approach that adds timestamped resampling over four evolutionary multiobjective algorithms and random matrix theory based filtering.

The combined approach was run on four filtered set of parameters. Out of these, three corresponded to the strategy based on Krzanowski stability suggested by Daly et al. and the fourth to the alternative introduced by Plerou et al.. The first three were specifically KR2, KR4 and KR8, the combination of parameters that offered the best results in previous financial portfolio optimization tasks. All of these reconstruction strategies were tested on a 20 -year return series on the components of the Spanish broad index, IBEX-35, using four core algorithms: two genetic algorithms NSGA-II and SPEA2, a representative of multiobjective differential evolution, GDE3, and a multiobjective particle swarm optimization algorithm, SMPSO.

The stability of the solutions was assessed based on four different metrics: stability, estimation error, sensitivity to extreme scenarios and unrealised returns. The use of the combined approach improved significantly the stability of the final solution regardless of the filtering chosen. Having said that, KR strategies, specially KR4 and KR8, clearly outperformed Plerou et al. The choice of the basic metaheuristic did no have any impact on that regard, as the mentioned pattern was very prevalent. It made, however, a big difference in terms of obtaining the most robust portfolios. No core algorithm offered better results than the rest across all of the four ro- bustness indicators, but NSGA-II turned out to be the dominant one. This genetic algorithm offered the best performance for ER, EE and ST. SMPSO provided the best results in terms of UR, as its portfolios tended to be closer to the actual efficient frontier. For this reason, unless the decision maker had a very special interest in UR, among the reviewed alternatives we would recommend NSGA-II.

Future extensions of this work might include the increase of range of core algorithms tested, a comprehensive optimization effort on the parameters of each algorithm or the analysis of the scalability of the approach with larger sets of investment alternatives.

\section{Acknowledgements}

The authors acknowledge financial support granted by the Spanish Ministry of Economy and Competitivity under grant ENE2014-56126-C2-2-R.

\section{References}

1. H.M. Markowitz, "Portfolio selection," The Journal of Finance, 7 (1):77-91 (1952).

2. H.M. Markowitz, Portfolio Selection: efficient diversification of investments. John Wiley \& Sons (1959).

3. H.J.C. Barbosa and A.C.C. Lemonge, "A genetic algorithm encoding for a class of cardinality constraints," Proceedings of the 2005 conference on Genetic and evolutionary computation, GECCO '05, 1193-1200, New York, NY, USA (2005)

4. K. Metaxiotis and K. Liagkouras. Multiobjective evolutionary algorithms for portfolio management: A comprehensive literature review," Expert Syst. Appl., 39 (14), 11685-11698 (2012).

5. P. Skolpadungket, K. Dahal, and N. Harnpornchai, "Portfolio optimization using multi-objective genetic algorithms. In Proceeding of 2007 IEEE Congress on Evolutionary Computation, 516-523 (2007).

6. I. Radziukyniene and A. Xilinskas, "Evolutionary methods for multi-objective portfolio optimization. In S. I. Ao, Len Gelman, David WL Hukins, Andrew Hunter, and A. M. Korsunsky, editors," Proceedings of the World Congress on Engineering 2008 Vol II, WCE '08, July 2 - 4, 2008, London, U.K., Lecture Notes in Engineering and Computer Science, pages 11551159. International Association of Engineers, Newswood Limited (2008).

7. K.P. Anagnostopoulos and G. Mamanis, "The meanvariance cardinality constrained portfolio optimiza- 
tion problem: An experimental evaluation of five multiobjective evolutionary algorithms," Expert Systems with Applications, 38 (11), 14208-14217 (2011).

8. K. Deb, R.E. Steuer, R. Tewari, and R. Tewari, "Biobjective portfolio optimization using a customized hybrid nsga-II procedure," EMO, 358-373 (2011).

9. F.J. Fabozzi, P.N. Kolm, Dessislava A. Pachamanova, and S.M. Focardi, "Robust Portfolio Optimization", The Journal of Portfolio Management, 33 (3), 40-48 (2007).

10. I. Paenke, J. Branke and Y. Jin, "Efficient Search for Robust Solutions by Means of Evolutionary Algorihtms and Fitness Approximations", IEEE Trans. on Ev. Comp., 10 (4), 405 - 420 (2006).

11. H.G. Beyer and B. Sendhoff, "Robust optimization - A comprehensive survey", Comput. Methods Appl. Mech. Engrg., 196, 33 - 34, 3190 - 3218 (2007).

12. O.L.V. Costa and A.C. Paiva, "Robust portfolio selection using linear-matrix inequalities", Journal of Economic Dynamics and Control, 26 (6), 889-909 (2002).

13. T.D. Nguyen, and A.W Lo, " Robust ranking and portfolio optimization”, European Journal of Operational Research, 221 (2), 407 - 416 (2012).

14. L. El Ghaoui, M. Oks and F. Oustry, "WorstCase Value-At-Risk and Robust Portfolio Optimization: A Conic Programming Approach", Oper. Res., INFORMS, 51 (4), 543-556 (2003).

15. S. Zymler, B. Rustem, and D. Kuhn, "Robust portfolio optimization with derivative insurance guarantees", European Journal of Operational Research, 210 (2), 410 - 424 (2011).

16. C. Perret-Gentil and M.-P. Victoria-Feser, "Robust mean-variance portfolio selection," Fame research paper series, International Center for Financial Asset Management and Engineering (2005).

17. G. Pflug and D. Wozabal, "Ambiguity in portfolio selection," Quantitative Finance, 7 (4), 435-442 (2007).

18. H. Shiraishi, "Resampling procedure to construct value at risk efficient portfolios for stationary returns of assets," ss (2008).

19. A. Grazia Quaranta and A. Zaffaroni, "Robust optimization of conditional value at risk and portfolio selection ", Journal of Banking \& Finance, 32 (10), 2046 - 2056 (2008).

20. V. Serbin, M. Borkovec, and M Chigirinskiy, "Robust Portfolio Rebalancing with Transaction Cost Penalty an Empirical Analysis", Journal of Investment Management (JOIM) , 9 (2), 73 - 87 (2011).

21. R.H. Tütüncü and M. Koenig, "Robust asset allocation," Annals OR, 132 (1-4), 157-187 (2004).

22. K. Deb and H. Gupta, "Introducing Robustness in Multi-Objective Optimization”, Evolutionary Computation,14 (4), 463 - 494 (2006)

23. S. García, D. Quintana, I.M. Galván, and P. Isasi, "Time-stamped resampling for robust evolutionary portfolio optimization," Expert Systems with Applications, 39 (12), 10722-10730 (2012).

24. V. Plerou, P. Gopkrishnan, B. Rosenow, L. A. N. Amaral, and H.E. Stanley, "A random matrix theory approach to financial cross-correlations," Physica A: Statistical Mechanics and its Applications, 287, 374382 (2000).

25. L. Laloux, P. Cizeau, J-P. Bouchaud, and M. Potters. Random matrix theory and financial correlations," International Journal of Theoretical and Applied Finance, 3 (3), 391-397 (2000).

26. S. Sharifi, M. Crane, A. Shamaie, and H. Ruskin, "Random matrix theory for portfolio optimization: a stability approach," Physica A: Statistical Mechanics and its Applications,335 (3), 629-643 (2004).

27. J. Daly, M. Crane, and H.J. Ruskin, " Random matrix theory filters in portfolio optimisation: A stability and risk assesment," Physica A: Statistical Mechanics and its Applications, 387, 4248-4260 (2008).

28. A. M. Sengupta and P. P. Mitra, "Distributions of singular values for some random matrices," Physical Review E, 60 (3), 3389-3392 (1999).

29. W.J. Krzanowski, "Sensitivity of principal components," Journal of the Royal Statistical Society. Series B (Methodological), 46 (3), 558-563 (1984).

30. K. Deb, A. Pratap, S. Agarwal, and T. Meyarivan, "A fast and elitist multiobjective genetic algorithm: Nsgaii," IEEE Transactions on Evolutionary Computation, 6 (2), 182-197 (2002).

31. E. Zitzler, M. Laumanns, and L. Thiele, "SPEA2: Improving the strength pareto evolutionary algorithm," Technical Report 103, Computer Engineering and Networks Laboratory (TIK), Swiss Federal Institute of Technology (ETH), Zurich, Switzerland, (2001).

32. A.J. Nebro, J.J. Durillo, J. García-Nieto, C.A. Coello Coello, F. Luna, and E. Alba, "Smpso: A new psobased metaheuristic for multi-objective optimization," 2009 IEEE Symposium on Computational Intelligence in Multicriteria Decision-Making (MCDM 2009), 6673. IEEE Press, (2009).

33. M. Clerc and J. Kennedy. "The particle swarm - explosion, stability, and convergence in a multidimensional complex space," IEEE Transactions on Evolutionary Computation, 6 (1), 58-73 (2002).

34. S. Kukkonen and J. Lampinen, "Gde3: The third evolution step of generalized differential evolution," IEEE Congress on Evolutionary Computation (CEC'2005), 443-450 (2005).

35. E. Zitzler and L. Thiele, "An evolutionary algorithm for multiobjective optimization: The strength pareto approach," Technical Report 43, ss, Gloriastrasse 35, CH-8092 Zurich, Switzerland (1998).

36. P.C. Mahalanobis, "On the generalized distance in statistics,” Proc. Nat. Inst. Sci., 2, 49-55 (1936).

37. J.J. Durillo, A.J. Nebro, and E. Alba. "The jmetal 
D. Quintana et al.

framework for multi-objective optimization: Design and architecture. In CEC 2010, volume 5467 of Lec- ture Notes in Computer Science, pages 4138-4325 (2010). 